Purdue University

Purdue e-Pubs

CTRC Research Publications

Cooling Technologies Research Center

2017

\title{
Characterization of Liquid Film Thickness in Slug- Regime Microchannel Flows
}

R. S. Patel

Purdue University

J.A. Weibel

Purdue University, jaweibel@purdue.edu

S V. Garimella

Purdue University, sureshg@purdue.edu

Follow this and additional works at: http://docs.lib.purdue.edu/coolingpubs

Patel, R. S.; Weibel, J. A.; and Garimella, S V., "Characterization of Liquid Film Thickness in Slug-Regime Microchannel Flows" (2017). CTRC Research Publications. Paper 319.

http://dx.doi.org/http://dx.doi.org/10.1016/j.ijheatmasstransfer.2017.08.008

This document has been made available through Purdue e-Pubs, a service of the Purdue University Libraries. Please contact epubs@purdue.edu for additional information. 


\title{
Characterization of Liquid Film Thickness in Slug-Regime Microchannel
}

\section{Flows}

\author{
Ravi S. Patel, Justin A. Weibel, and Suresh V. Garimella ${ }^{1}$ \\ Cooling Technologies Research Center, an NSF IUCRC \\ School of Mechanical Engineering and Birck Nanotechnology Center \\ Purdue University, West Lafayette, IN 47907-2088 USA
}

\begin{abstract}
An experimental investigation is conducted to examine the effects of operating conditions and channel size on the liquid film thickness of vapor bubbles in adiabatic air-water flows within the slug flow regime. Acrylic test sections are fabricated to contain a single microchannel of square cross-section with hydraulic diameters of $510 \mu \mathrm{m}$ and $1020 \mu \mathrm{m}$. High-speed visualizations are used to map the flow regimes in these channels in order to determine the range of liquid and gas flow rates for which slug-regime flows are sustained. Subsequently, a tomographic optical imaging technique is employed to quantitatively reconstruct the liquid-gas interface of vapor bubbles at selected operating conditions. This technique relies on visualization of fluorescent particles seeded into the liquid phase in order to identify the phase boundaries within thin sections of the flow. Using the reconstructions, the thickness of the liquid film in the corner of the square channel cross-section is extracted. This film thickness is found to decrease with increasing capillary number, and a simple expression is proposed for calculation of the film thickness; predictions from this model match the measurements with a mean absolute error (MAE) of 21.6\%; $85.7 \%$ of all predicted data points fall within an error band of $\pm 30 \%$. Additionally, film thickness data from the literature are compared to model predictions and a comparable MAE of $21.8 \%$ is found with $77.8 \%$ of data points falling within an error band of $\pm 30 \%$.
\end{abstract}

\footnotetext{
${ }^{1}$ Author to whom correspondence should be addressed: (765) 494-6209, sureshg@purdue.edu
} 


\section{KEYWORDS}

two-phase microchannel flow; slug regime; liquid film thickness; segmented flow

\section{NOMENCLATURE}

$\mathrm{Ca} \quad$ capillary number $(\mu u / \sigma)$

Co confinement number

$$
\left(\left(\sigma / g\left(\rho_{f}-\rho_{g}\right)\right)^{0.5} / D_{H}\right)
$$

$N A \quad$ numerical aperture

$D_{f} \quad$ depth of field, $\mathrm{m}$

$D_{H} \quad$ hydraulic diameter, $\mathrm{m}$

$j \quad$ superficial phase velocity, $\mathrm{m} \mathrm{s}^{-2}$

$u \quad$ velocity, $\mathrm{m} \mathrm{s}^{-1}$

$x \quad$ streamwise coordinate position, $\mathrm{m}$

y spanwise coordinate position, $\mathrm{m}$

$z \quad$ depth coordinate position, $\mathrm{m}$

\section{Greek Symbols}

$\alpha \quad$ volumetric void fraction

$\delta \quad$ corner film thickness, $\mathrm{m}$

$\mu \quad$ dynamic viscosity, $\mathrm{kg} \mathrm{m}^{-1} \mathrm{~s}^{-1}$

$\rho \quad$ density, $\mathrm{kg} \mathrm{m}^{-3}$

$\sigma \quad$ surface tension, $\mathrm{N} \mathrm{m}^{-1}$

\section{Subscripts}

$f \quad$ liquid phase

$g \quad$ gas phase

\section{INTRODUCTION}

The slug flow regime is commonly encountered in small-scale channels [1] owing to the significant influence of surface tension on the flow morphology [2]. This slug flow regime is characterized by elongated bubbles occupying the width of the channel that are separated by liquid slugs in the streamwise direction. Several distinct benefits to the slug flow regime result from this unique morphology; specifically, heat and mass transfer processes benefit from the high gas-phase surface-to-volume ratio [1], enhanced mixing, and presence of very thin liquid films [3], thus 
offering advantages relative to other flow regimes in chemical processing, thermal management and other applications. Despite these advantages, quantitative investigation of liquid-gas interface geometries in the slug flow regime has been limited mainly by the lack of suitable metrological techniques [4]. Characterization of the liquid film structure would aid in improved performance prediction capabilities that would benefit device design.

Jacobi and Thome [5] developed a mechanistic model to predict the wall heat transfer coefficient in circular microchannels operating in the slug flow regime based on an assumption that thin film evaporation was the predominant heat transfer mechanism. Their two-zone model represented the flow as a cyclical passage of a liquid slug zone followed by a two-phase region wherein the channel walls were uniformly coated by an evaporating liquid film. The film thickness was left as a free input parameter. A subsequent update was introduced by Thome et al. [6] in order to reduce the number of free parameters and to represent the actual flow conditions with higher fidelity. This three-zone model added a third, dryout region trailing the two-phase region, and included an initial film thickness in the two-phase region computed using the correlation of Moriyama and Inoue [7] that accounts for a thinning of the liquid film along the bubble length based on an energy balance. The film thickness correlation was originally developed for bubble growth between parallel plates, and was based on calculated film thickness estimates rather than direct measurements of the film [7]; it was modified by Thome et al. [6] to provide a better fit to the experimental data obtained in a microchannel flow environment. Harirchian and Garimella [8] subsequently applied the three-zone model to a large set of experimental heat transfer coefficient measurements and calculated the initial film thickness using an alternative correlation presented by Aussillous and Quere [9]. This correlation was originally developed through visualization of the film deposited by a single liquid plug on the walls of capillary tubes; it was modified by 
Harirchian and Garimella [8] in order to maximize the accuracy of the predictions yielded by the three-zone model. Recently Magnini and Thome [10] published another update to the three-zone model, which included the evaluation of several experimental liquid film thickness correlations obtained in circular capillary tubes.

Current mechanistic models for microchannel slug flows have relied on estimates of the liquid film thickness and measurements acquired in flow environments that are quite different from the application of interest, and thereby have had to employ correction factors to improve model performance. Detailed information specific to vapor bubble geometry and liquid film thickness in microchannel slug flows has not been reported to date. Tibirica et al [4] presented an exhaustive review of extant metrological techniques targeted towards thin film measurements and attributed this lack of film characterization to difficulties associated with performing measurements at a sufficiently high speed in a non-intrusive manner. Han and Shikazono [11] successfully deployed laser focus displacement meters in the investigation of liquid film thickness at the channel sidewall and corner in microchannels of square cross-section ranging in hydraulic diameter of $0.3 \mathrm{~mm}$ to 1 mm. The liquid film cross-section was described as being either axisymmetric and circular in shape, or non-axisymmetric and made up of line segments parallel to the channel walls joined by quarter-circle arcs at the corners. For cases where cross-sectional film profiles were nonaxisymmetric, a sidewall film of constant thickness was observed [11]. This thickness was found to be independent of operating conditions and was sufficiently thin that it was assumed as having a ratio of film thickness to hydraulic diameter of nearly zero. In the experiments of Han and Shikazono [11], slug flow was mimicked by drawing out liquid from an initially flooded channel with the opposite end open to air, yielding an extended air bubble surrounded by a liquid film that is pulled into the channel. Data gathered during this study were used to develop a correlation for 
the sidewall and corner film thicknesses. Fries et al. [12] demonstrated the ability to resolve the cross-sectional liquid film profile in a true microchannel slug flow environment using laserinduced fluorescence of a dye incorporated into the liquid phase, which was visualized in a tomographic fashion with confocal scanning microscopy. The sidewall film thickness was found to have a constant value with the ratio of film thickness to hydraulic diameter being 0.02 . The primary limitation of the technique was the low temporal resolution of the confocal microscope, which only yielded a highly time-averaged film thickness measurement that could not resolve the streamwise-varying nature of slug flows. Chaoqun et al. [13] applied a more straightforward and simplified approach by performing high-speed visualizations of slug flows in a channel of $750 \mu \mathrm{m}$ $\times 280 \mu \mathrm{m}$ cross-section. Film thickness was deduced from visualizations obtained through the wider face of the channel by assuming that the flat regions of the bubble observed against the channel walls were joined by a circular arc. In this manner, a corner film thickness was calculated for individual cases, but no model or correlation was proposed based on the data. In general, experimental measurements of the very small sidewall film thickness suffer from comparatively large uncertainties [11,12]; numerical simulations of semi-infinite bubbles such as those characterized by Han and Shikazono [11] revealed a fixed ratio of sidewall film thickness to hydraulic diameter of 0.01 [14].

The present work seeks to characterize the liquid film thickness in slug-flow regime microchannel flows based on a parametric experimental investigation. Adiabatic two-phase flow experiments are performed in channels of square cross-section with hydraulic diameters of 510 $\mu \mathrm{m}$ and $1020 \mu \mathrm{m}$ over a range of liquid and gas phase flow rates. The film thickness is measured utilizing an experimental approach previously developed by the authors [15] to quantitatively reconstruct the three-dimensional liquid-gas interface shape of bubbles within slug-regime 
microchannel flows. Once film measurements are obtained, a model for the corner liquid film thickness is developed. As outlined above, this corner film thickness is more variable than the sidewall film thickness and plays a more significant role in determining the overall cross-sectional liquid film profile. It can also be characterized experimentally to a greater degree of accuracy. The predictive performance of the model is benchmarked against both data obtained in the present study as well as film thickness measurements available in the literature.

\section{EXPERIMENTAL METHODS}

\subsection{Experimental Flow Loop}

The experimental facility shown in Figure 1 is briefly described here; additional details are available in Patel et al. [16]. The schematic diagram in Figure 1b illustrates the different flow paths in the loop. The closed liquid loop contains a primary line throttled with a needle valve, and a metered secondary line tapped from the primary loop to feed liquid to the horizontally oriented microchannel test section at a constant pressure. The measurement uncertainties are $\pm 1 \%$ of full scale (FS) (Omega, FLR1007-D) and $\pm 2 \%$ FS (Alicat, LC-10CCM-D) for the metering in this secondary line. In the open gas loop pressurized air is throttled with a needle valve, metered, injected into the channel, and subsequently separated at the reservoir. The gas flow meters each have a measurement uncertainty of $\pm 1.5 \%$ FS (Omega, part nos. FMA3105 and FMA3102).

Two test sections of different microchannel sizes are used for the experiments. Each test section contains a single, saw-cut microchannel of square cross-section with a hydraulic diameter of $510 \mu \mathrm{m}$ or $1.02 \mathrm{~mm}$. Adiabatic two-phase flows are generated in the channel through the controlled injection of air into a liquid flow at a T-junction upstream of an observation location 
where optical characterization of the liquid film is performed. A flow length of 100 diameters along the channels between the T-junction and the observation location ensures full development of the two-phase flow. The working liquid is water seeded with $0.5 \mu \mathrm{m}$-diameter fluorescent polystyrene microspheres (Magsphere Inc., PSF-500NM) at a concentration of $0.025 \%$ volume fraction; the working gas is compressed air. At the concentration used, the presence of these particles has a negligible impact on the density, viscosity [17,18], and surface tension [19,20].

\subsection{Imaging and Interface Characterization}

Vapor bubble interface shapes are characterized based on the rectilinear coordinate system identified in Figure 2. The corner liquid film thickness, $\delta$, is taken as the distance along the channel diagonal from the channel corner to the liquid-gas interface, and is measured at the streamwisemidpoint along the bubble length. This is done to minimize the impact of any local flow effects near the leading and trailing bubble caps on the film thickness. In the case of a long adiabatic vapor bubble, exceeding several hydraulic diameters, a region exists wherein the cross-sectional interface profile does not change along the streamwise direction, and the corner film thickness measurement remains constant. For these cases, the corner film measurement can be obtained anywhere along this fully developed length.

The liquid-gas interface of the bubbles is characterized using a tomographic optical imaging method previously described by the authors [15]. This method leverages the relatively shallow depth-of-field of the objective lenses to visualize the seeding particles within thin sections of the flow, and is briefly described here. Texture-based image processing techniques are employed in order to resolve boundaries between the liquid phase, which contains the seeding 
particles, and the gas phase with an accuracy of $\pm 2.8 \mu \mathrm{m}$ at a magnification of $10 \times$ or $\pm 6.7 \mu \mathrm{m}$ at a magnification of $4 \times[15]$. The resulting accuracy of corner film thickness measurements considered in the present study ranges from $2.5 \%$ to $6.8 \%$; for the purpose of developing a model, the film thickness is most accurately characterized in this corner location (compared to the much thinner sidewall film). The visualizations are obtained through an inverted epifluorescent optical microscope (Nikon Ti-U) with illumination delivered by a $5 \mathrm{~ns}$ pulsed laser having a wavelength of $532 \mathrm{~nm}$, enabling the capture of high-quality still images of the flow. A 10x objective lens (Nikon CFI Plan Fluor 10x) with a numerical aperture, NA, of 0.30 and estimated focal plane thickness, $D_{f}$, of $7.1 \mu \mathrm{m}$ was used to characterize flows within the smaller channel size ( $D_{H}=510$ $\mu \mathrm{m}$ ), and a $4 \times$ objective lens (Nikon CFI Plan Fluor $4 \times, N A=0.13, D_{f}=39.8 \mu \mathrm{m}$ ) was used for the larger channel size $\left(D_{H}=1020 \mu \mathrm{m}\right)$. Fluorescent images are recorded with a monochrome CCD camera (Photometrics CoolSNAP HQ) with a sensor resolution of $1392 \times 1040$ pixels and a pixel pitch of $6.45 \mu \mathrm{m}$. Separately, the microscope is configured to perform standard optical imaging using a high-speed camera (Photron FASTCAM 1024PCI) for the purpose of flow regime identification.

Imaging is performed at discrete depths within the lower half of the channel cross-section and the bubbles are assumed to be unaffected by gravity (i.e., vertically symmetric) based on a confinement-number transition criterion from the literature [21]. Ong and Thome [21] performed visualizations to investigate gravitational effects on flow morphology in small-scale channels and found that flows with Co greater than 1 were unaffected by buoyancy forces. For the two test sections in the present study, Co $=2.7$ for $D_{h}=1020 \mu \mathrm{m}$ and Co $=5.4$ for $D_{h}=510 \mu \mathrm{m}$. 


\section{RESULTS}

\subsection{Flow Regime Mapping}

A flow regime mapping was performed in each test section using high-speed visualizations in order to determine the operating conditions necessary to generate slug-regime flows. The resulting maps are shown in Figure 3a-b and representative images of the flows within each region of the map are presented in Figure 3c; these images were obtained in the $D_{H}=510 \mu \mathrm{m}$ test section. The superficial gas-phase velocity, $j_{g}$, is plotted on the horizontal axis and the superficial liquidphase velocity, $j_{f}$, is plotted on the vertical axis. The three primary flow regimes observed are: (1) bubbly flow, featuring unconfined gas bubbles with diameters smaller than the channel width; (2) slug flow; and (3) annular flow, featuring a continuous vapor core and a thin liquid film along the channel walls. Additional transitional morphologies separating the primary flow regimes are also observed, such as an intermittent churn flow at the slug-annular transition. The test section with a larger channel size shows a greater prevalence of unconfined bubbly flows whereas the smaller channel size has a much smaller proportion of the regime map occupied by bubbly flows and a greater tendency to operate in a slug flow regime.

\subsection{Interface Characterization and Modeling}

Representative vapor bubble interfaces are shown in Figure 4 for three selected test cases from the test section with the larger channel size; the operating conditions for each test case are outlined in Table 1. The measured interface profiles at varying $z$ positions within the channel resulting from the tomographic characterization approach are shown on the left of the figure, while three-dimensional reconstructions are shown on the right. The measured profiles are reflected 
about the horizontal midplane of the channel assuming vertical symmetry as justified earlier, and a surface is fitted using cubic interpolation in order to generate the full interface reconstructions. Comparing Case 1 to Case $3, j_{f}$ is held fixed while $j_{g}$ is doubled by injecting more air into the flow, yielding a longer bubble length. From Case 2 to Case $3, j_{g}$ is held fixed while $j_{f}$ is halved by reducing the liquid pumped into the channel, again yielding a longer bubble. The volumetric void fraction, defined as $\alpha=j_{g} /\left(j_{g}+j_{f}\right)$, is held approximately constant from Case 1 to Case 2 while the volumetric flow rates of both phases are doubled to achieve the operating conditions at Case 2. The overall bubble lengths are measured as $2.18 \mathrm{~mm}$ for Case 1, $2.90 \mathrm{~mm}$ for Case 2, and 3.65 $\mathrm{mm}$ for Case 3 . This increase in length of only $33 \%$ between Case 1 and 2 reveals that the bubble generation frequency increased between these operating points, as the increase in shear stress from the higher liquid flow rate induces earlier bubble departure from the T-junction. Case 1 has the lowest capillary number of the three cases presented in Figure 4. For this case $\left(\mathrm{Ca}=6.75 \times 10^{-3}\right)$, the rear cap is hemispherical in shape and grows less convex with increasing $\mathrm{Ca}$ for Case $3(\mathrm{Ca}=$ $\left.1.15 \times 10^{-2}\right)$ and Case $2\left(\mathrm{Ca}=1.37 \times 10^{-2}\right)$. This decrease in rear cap convexity arises from the increase in liquid recirculation behind the vapor bubble and the higher interfacial shear stresses acting on the bubble. Findings in the literature have identified similar trends and concluded that at a Ca value of $2.0 \times 10^{-2}$ the rear cap will flatten completely [22]. The characteristic velocity for the calculation of $\mathrm{Ca}$ is taken as the average flow velocity, $u=j_{g}+j_{f}$.

Tomographic fluorescent imaging is performed at all test points shown with open symbols in Figure 3 in order to extract corner film thickness values. These film thicknesses are subsequently nondimensionalized by normalizing to the hydraulic diameter of the channel in which they were measured. Figure 5 plots the normalized values as a function of capillary number. Based on the liquid and gas flow-rate metering accuracies discussed in Section 2.1, the uncertainty 
in $\mathrm{Ca}$ over all cases considered ranges from $1.2 \%$ to $7.4 \%$, as found using a standard uncertainty analysis procedure [23]. In general, Figure $\mathbf{5}$ shows that nondimensional film thickness decreases with increasing $\mathrm{Ca}$ values. However, an outlier at $\mathrm{Ca}=2.26 \times 10^{-2}$ is found to have a film thickness greater than the other points at this capillary number due to its proximity to the bubbly-to-slug transition region of unconfined flow shown in Figure 3a $\left(D_{H}=1020 \mu \mathrm{m}, j_{g}=0.66 \mathrm{~m} / \mathrm{s}, j_{f}=1.0\right.$ $\mathrm{m} / \mathrm{s}$ ). Such operating conditions can promote detachment of the film from the channel walls. Using a linear regression, the following expression is developed to calculate normalized corner film thickness as a function of capillary number:

$$
\frac{\delta}{D_{H}}=0.085 \mathrm{Ca}^{-0.137}
$$

The functional form of the expression was chosen based on the importance of viscosity and surface tension effects in governing slug-regime flow morphology. Previous publications have presented expressions of similar form for macroscale flows in tubes [24,25], with the coefficients presented here fitted to predict film properties in a microchannel flow environment.

The performance of this film thickness model is assessed in Figure 6a, where experimental measurements are plotted on the horizontal axis and model predictions are plotted on the vertical axis. The experimental data are predicted with a mean absolute error (MAE) of $21.6 \%$, and 85.6\% of all predictions fall within an error band of $\pm 30 \%$. The model performance is also benchmarked against the experimental data of Chaoqun et al. [13] in Figure 6b, and successfully predicts film thickness with an MAE of $21.8 \% ; 77.8 \%$ of all predictions fall within an error band of $\pm 30 \%$. For this comparison, film thicknesses taken at $\mathrm{Ca}$ values in excess of 0.01 in the original study have been omitted; beyond this threshold, the higher aspect ratio of the channel showed strong inertial effects leading to liquid pooling in the narrow ends of the channel [13]. The film thickness 
measurements from the literature were obtained using an approach that relied on significant simplifying assumptions regarding bubble interface geometry, which may be responsible for the discrepancies in the comparison of the present model and the experimental data of Chaoqun et al. [13]; experimental uncertainties were not provided for the data. It is important to note that no additional data for possible comparison are available in the literature specifically for actively pumped two-phase flows. All other previous studies of the film thickness have simplified the flow configuration by studying a single bubble generated by evacuating liquid from an initially flooded channel [11], obtained limited data sets by estimating film thickness indirectly from visualizations under simplifying assumptions [22], or utilized measurement techniques that do not feature the requisite temporal resolution to successfully capture the streamwise-varying structures found in slug-regime flows [12].

\section{CONCLUSIONS}

A parametric experimental characterization of the liquid film thickness is performed in adiabatic slug-regime microchannel flows. The microchannel test sections considered had a square channel cross-section and hydraulic diameters of $510 \mu \mathrm{m}$ or $1020 \mu \mathrm{m}$. Flow regime mapping was performed using high-speed visualizations, which enabled the selection of operating points spanning a wide range of liquid and gas phase flow rates within the desired slug-flow regime for further testing. At the chosen interrogation points a tomographic optical imaging technique was employed in order to visualize fluorescent seeding particles incorporated in the liquid phase. The particles aided in distinguishing the phases and allowed for the quantitative identification of phase boundaries in discrete imaging planes with an accuracy better than $1 \%$ of overall channel size; the 
resulting accuracy in experimental corner film thickness measurements ranged from $2.5 \%$ to $6.8 \%$. Using this approach, interface geometries were reconstructed in three dimensions, from which corner film measurements could be extracted. The data were used to develop an empirical expression for the film thickness in terms of capillary number that reflects the observed decrease in film thickness with increasing Ca values. Assessment of model performance in predicting experimental measurements revealed an MAE of $21.7 \%$ with $82.1 \%$ of predictions falling within an error band of $\pm 30 \%$.

\section{ACKNOWLEDGEMENTS}

Financial support for this work provided by the Cooling Technologies Research Center, a National Science Foundation Industry/University Cooperative Research Center at Purdue University, is gratefully acknowledged.

\section{REFERENCES}

[1] S. Haase, T. Bauer, New method for simultaneous measurement of hydrodynamics and reaction rates in a mini-channel with Taylor flow, Chemical Engineering Journal 176-177 (2011) 65-74.

[2] H.C. Chang, M. Gad-el-Hak, The MEMS Handbook. CRC Press, Boca Raton, 2002.

[3] M.T. Kreutzer, F. Kapteijn, J.A. Moulijn, J.J. Heiszwolf, Multiphase monolith reactors: Chemical reaction engineering of segmented flow in microchannels, Chemical Engineering Science 60 (22) (2005) 5895-5916.

[4] C.B. Tibirica, F.J. Nascimento, G. Ribatski, Film thickness measurement techniques applied to micro-scale two-phase flow systems, Experimental Thermal \& Fluid Science 34 (4) (2010) 463-473. 
[5] A.M. Jacobi, J.R. Thome, Heat transfer model for evaporation of elongated bubble flows in microchannels, Journal of Heat Transfer 124 (6) (2002) 1131-1137.

[6] J.R. Thome, V. Dupont, A.M. Jacobi, Heat transfer model for evaporation in microchannels. Part I: presentation of the model, International Journal of Heat and Mass Transfer 47 (14-16) (2004) 3375-3385.

[7] K. Moriyama, A. Inoue, Thickness of the liquid film formed by a growing bubble in a narrow gap between two horizontal plates, Journal of Heat Transfer 118 (1) (1996) 132140.

[8] T. Harirchian, S.V. Garimella, Flow regime-based modeling of heat transfer and pressure drop in microchannel flow boiling, International Journal of Heat and Mass Transfer 55 (4) (2012) 1246-1260.

[9] P. Aussillous, D. Quere, Quick deposition of a fluid on the wall of a tube, Physics of Fluids 12 (10) (2000) 2367-2371.

[10] M. Magnini, J.R. Thome, An updated three-zone heat transfer model for slug flow boiling in microchannels, International Journal of Multiphase Flow 91 (2017) 296-314.

[11] Y. Han, N. Shikazono, Measurement of liquid film thickness in micro square channel, International Journal of Multiphase Flow 35 (10) (2009) 896-903.

[12] D.M. Fries, F. Trachsel, P.R. von Rohr, Segmented gas-liquid flow characterization in rectangular microchannels, International Journal of Multiphase Flow 34 (12) (2008) 11081118.

[13] Y. Chaoqun, Z. Yuchao, Y. Chunbo, D. Minhui, D. Zhengya, C. Guangwen, Characteristics of slug flow with inertial effects in a rectangular microchannel, Chemical Engineering Science 95 (2013) 246-256. 
[14] A.L. Hazel, M. Heil, The steady propagation of a semi-infinite bubble into a tube of elliptical or rectangular cross-section, Journal of Fluid Mechanics 470 (2002) 91-114.

[15] R.S. Patel, J.A. Weibel, S.V. Garimella, An optical approach for quantitative characterization of slug bubble interface profiles in a two-phase microchannel flow, International Journal of Heat and Mass Transfer 86 (2015) 31-38.

[16] R.S. Patel, J.A. Weibel, S.V. Garimella, Mechanistic modeling of the liquid film shape and heat transfer coefficient in annular-regime microchannel flow boiling, International Journal of Heat and Mass Transfer 114 (2017) 841-851.

[17] A. Einstein, Eine neue bestimmung der moleküldimensionen, Annalen der Physik 324 (2) (1906) 289-306.

[18] R. Roscoe, The viscosity of suspensions of rigid spheres, British Journal of Applied Physics 3 (8) (1952) 267-269.

[19] T. Okubo, Surface tension of structured colloidal suspensions of polystyrene and silica spheres at the air-water interface, Journal of Colloid and Interface Science 171 (1) (1995) $55-62$.

[20] B.P. Binks, Particles as surfactants—similarities and differences, Current Opinion in Colloid \& Interface Science 7 (1-2) (2002) 21-41.

[21] C.L. Ong, J.R. Thome, Macro-to-microchannel transition in two-phase flow: Part 2-Flow boiling heat transfer and critical heat flux, Experimental Thermal and Fluid Science 35 (6) (2011) 873-886.

[22] S. Haase, Characterisation of gas-liquid two-phase flow in minichannels with co-flowing fluid injection inside the channel, part II: Gas bubble and liquid slug lengths, film thickness, 
and void fraction within Taylor flow, International Journal of Multiphase Flow 88 (2017) 251-269.

[23] H.W. Coleman, W.G. Steele, Experimentation validation and uncertainty analysis for engineers, third ed., Wiley, Hoboken, 2009, pp. 61-81.

[24] F. Fairbrother, A.E. Stubbs, Studies in electro-endosmosis. Part VI. The bubble-tube method of measurement, Journal of the Chemical Society (Resumed) (1935) 527-529.

[25] F.P. Bretherton, The motion of long bubbles in tubes, Journal of Fluid Mechanics 10 (2) (1961) 166-188. 
Table 1 Operating conditions for the vapor bubble reconstructions presented in Figure 4.

\begin{tabular}{ccccc}
\hline $\begin{array}{c}\text { Case } \\
\text { No. }\end{array}$ & $\begin{array}{c}\text { Superficial } \\
\text { Liquid-Phase } \\
\text { Velocity } \\
\boldsymbol{j}_{\boldsymbol{f}}(\mathbf{m} / \mathbf{s})\end{array}$ & $\begin{array}{c}\text { Superficial } \\
\text { Gas-Phase } \\
\text { Velocity } \\
\boldsymbol{j}_{\boldsymbol{g}}(\mathbf{m} / \mathbf{s})\end{array}$ & $\begin{array}{c}\text { Volumetric } \\
\text { Void } \\
\text { Fraction } \\
\boldsymbol{\alpha}\end{array}$ & $\begin{array}{c}\text { Capillary } \\
\text { Number } \\
\mathbf{C a}\end{array}$ \\
\hline 1 & 0.16 & 0.33 & 0.67 & $\begin{array}{c}6.75 \times 10^{-} \\
3\end{array}$ \\
2 & 0.34 & 0.67 & 0.66 & $\begin{array}{c}1.37 \times 10^{-} \\
2\end{array}$ \\
3 & 0.16 & 0.67 & 0.81 & $\begin{array}{c}1.15 \times 10^{-} \\
2\end{array}$ \\
\hline
\end{tabular}



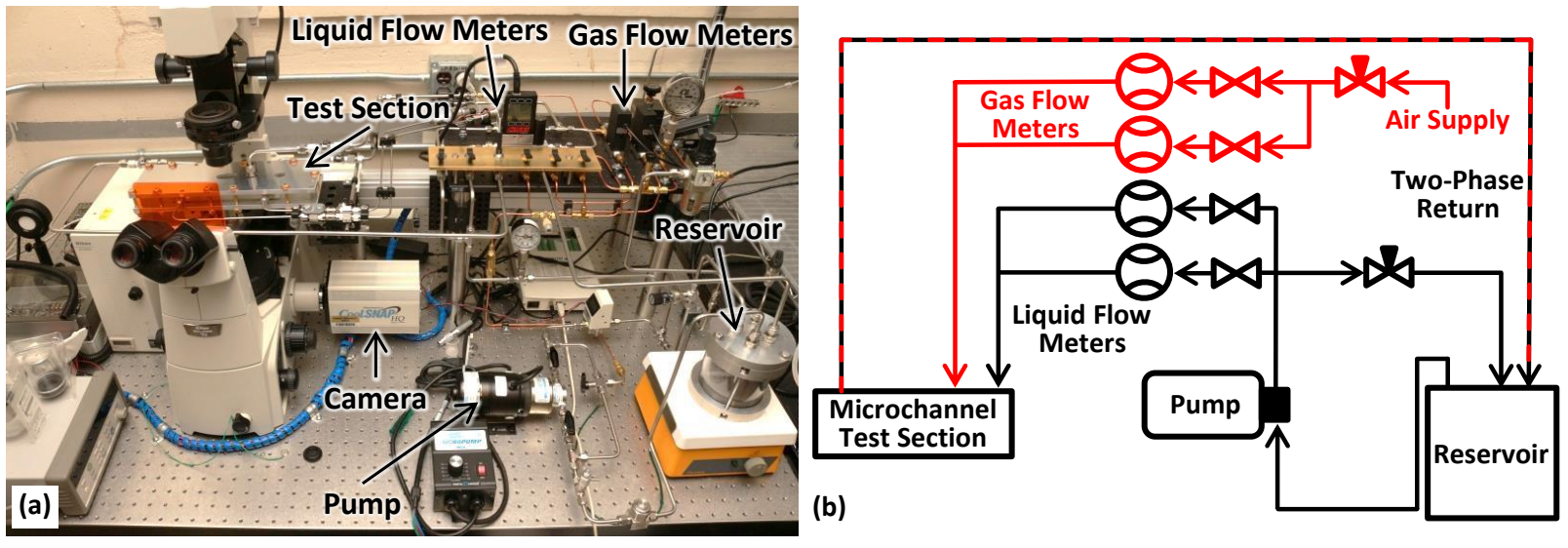

Figure 1 (a) Photograph of the experimental facility, and (b) schematic diagram of the flow loop with liquid lines shown in black and gas lines shown in red (Patel et al. 2017). 


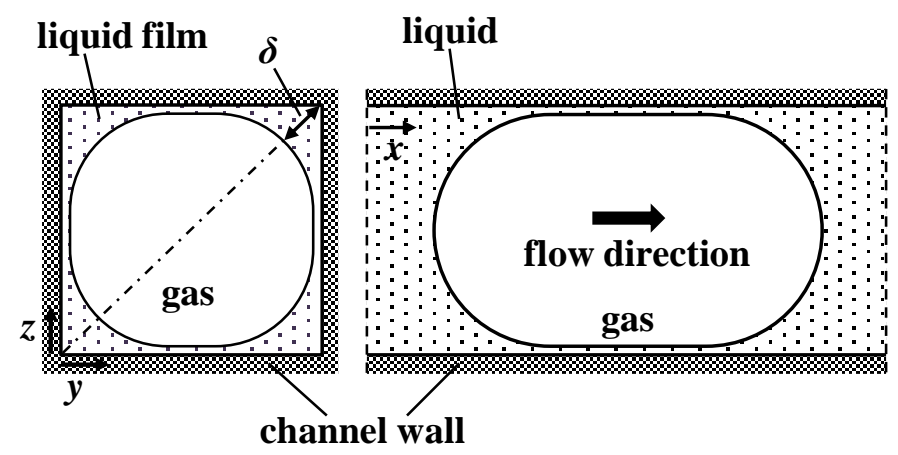

Figure 2 Schematic diagram of slug flow morphology and coordinate system used to describe liquid-gas interface shape within the channel cross-section (left) and streamwise direction (right). 


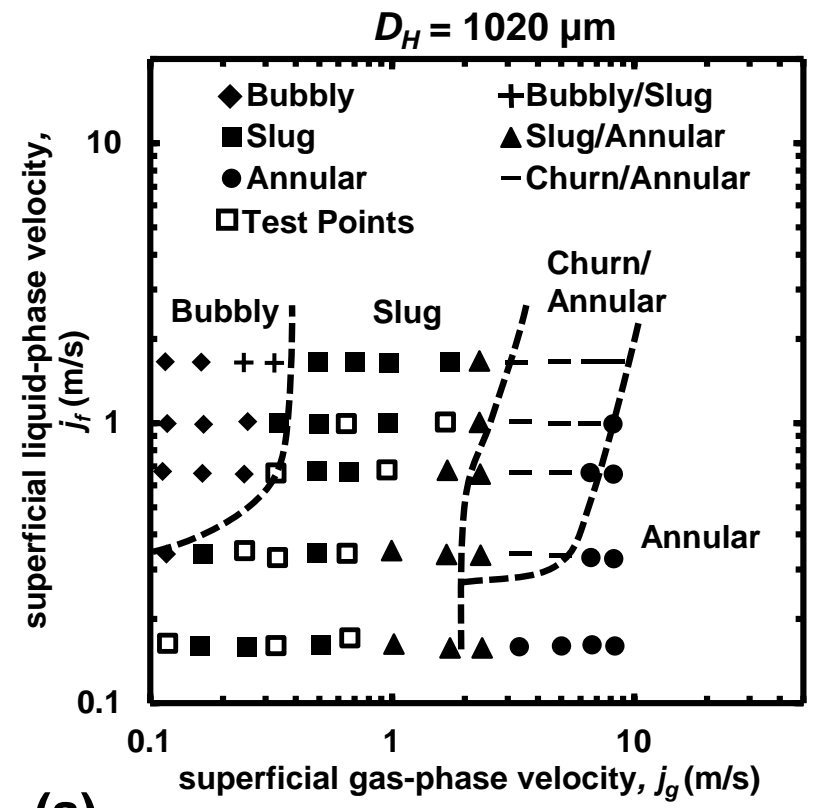

(a)

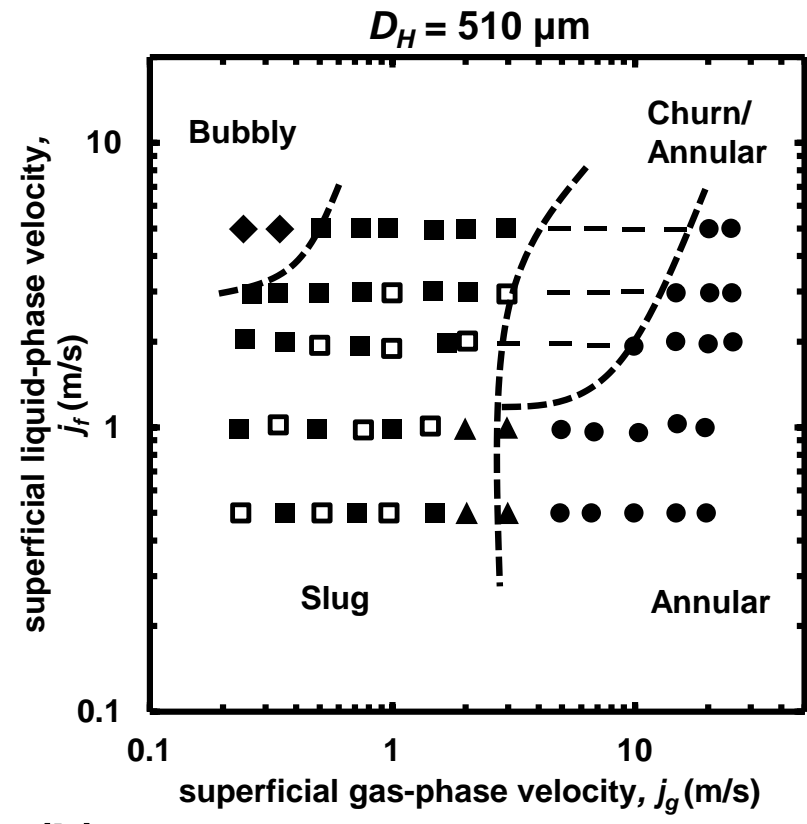

(b)

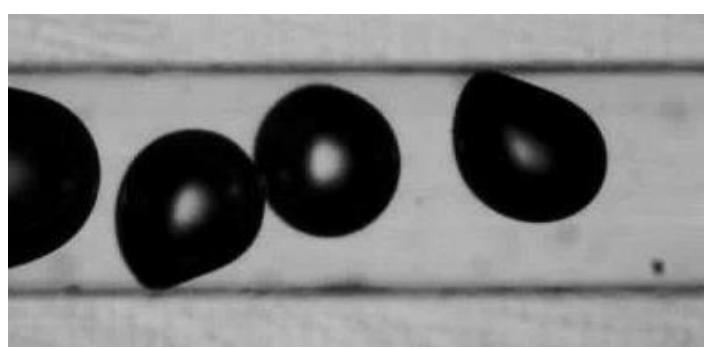

\section{Bubbly Flow}

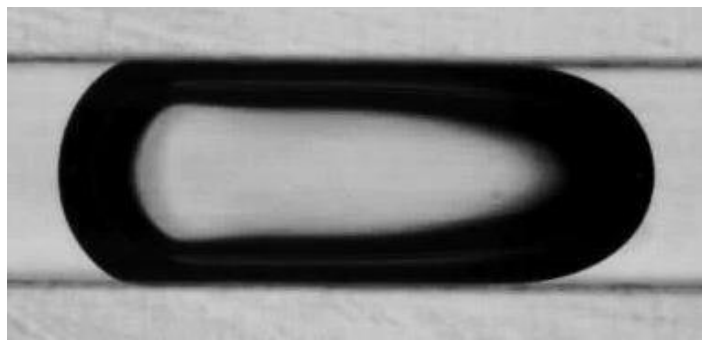

Slug Flow

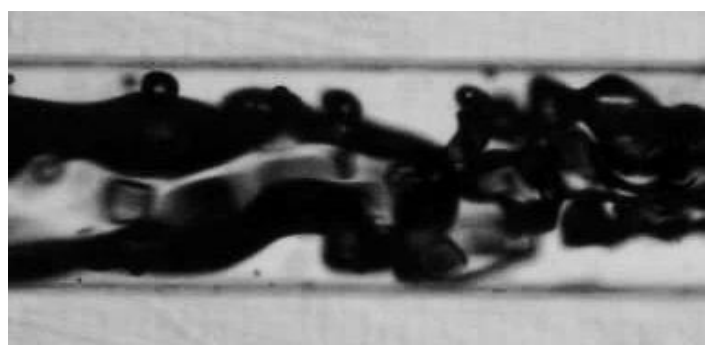

Churn Flow

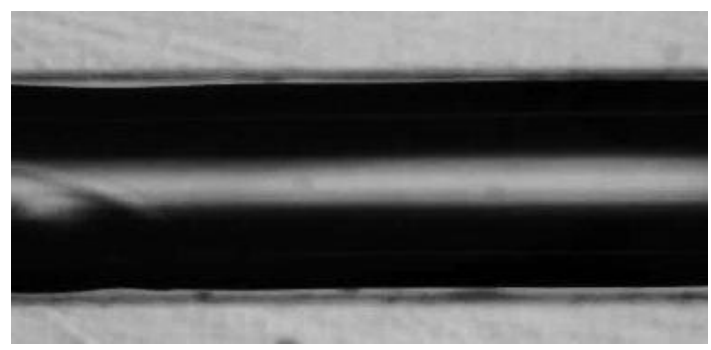

(c)

\section{Annular Flow}

Figure 3 Flow regime maps for the (a) $D_{H}=1020 \mu \mathrm{m}$ and (b) $D_{H}=510 \mu \mathrm{m}$ microchannel test sections. Operating points at which film characterization is performed are shown with open symbols, and (c) images taken in the $D_{\mathrm{H}}=510 \mu \mathrm{m}$ test section that are representative of the flow morphology for each operating region are identified. 

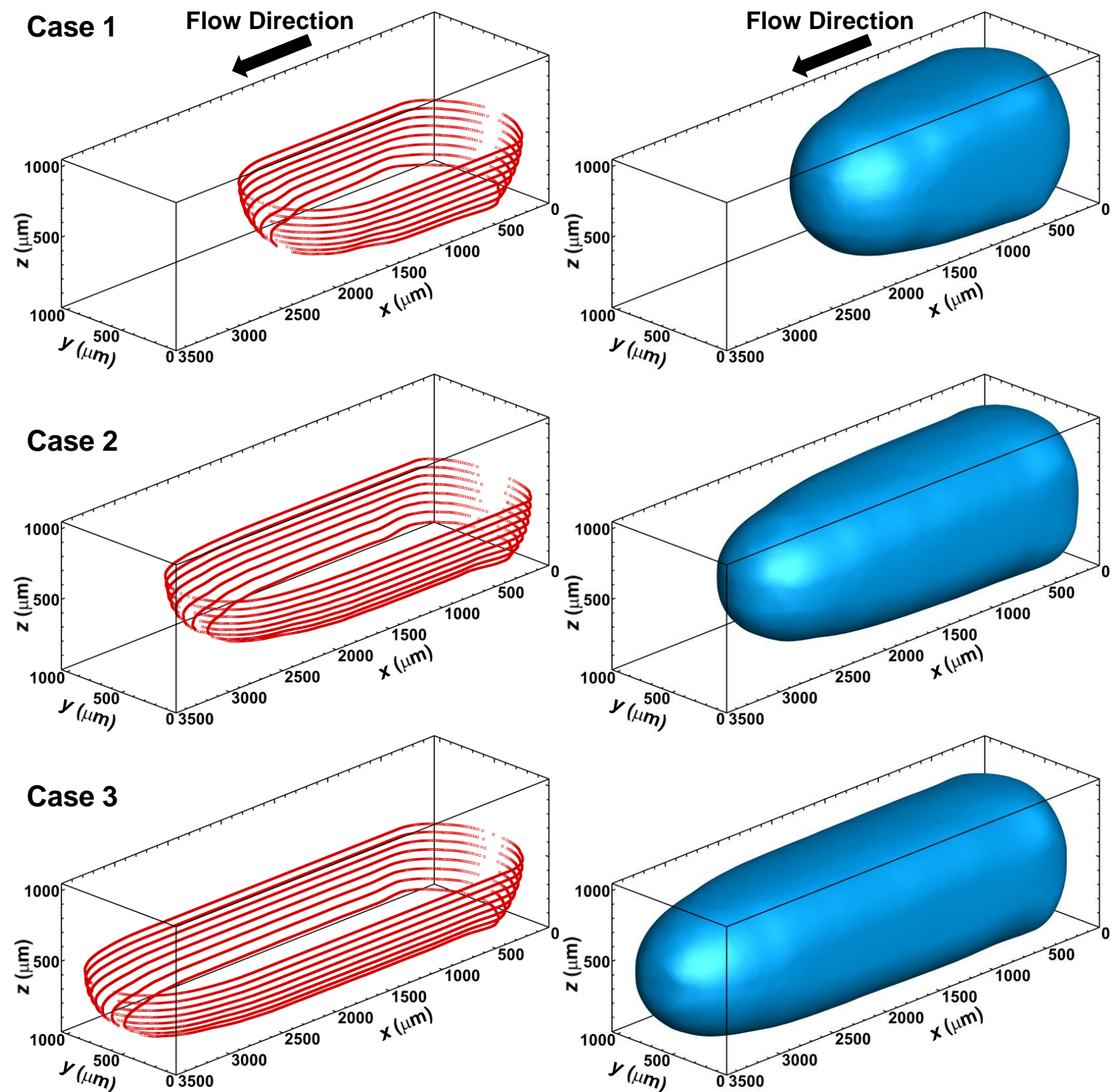

Figure 4 Representative interface characterization data taken from the $D_{H}=1020 \mu \mathrm{m}$ microchannel test section showing tomographic liquid-gas interface profiles at varying $z$ positions within the channel (left) and three-dimensional vapor bubble interface reconstructions (right). 


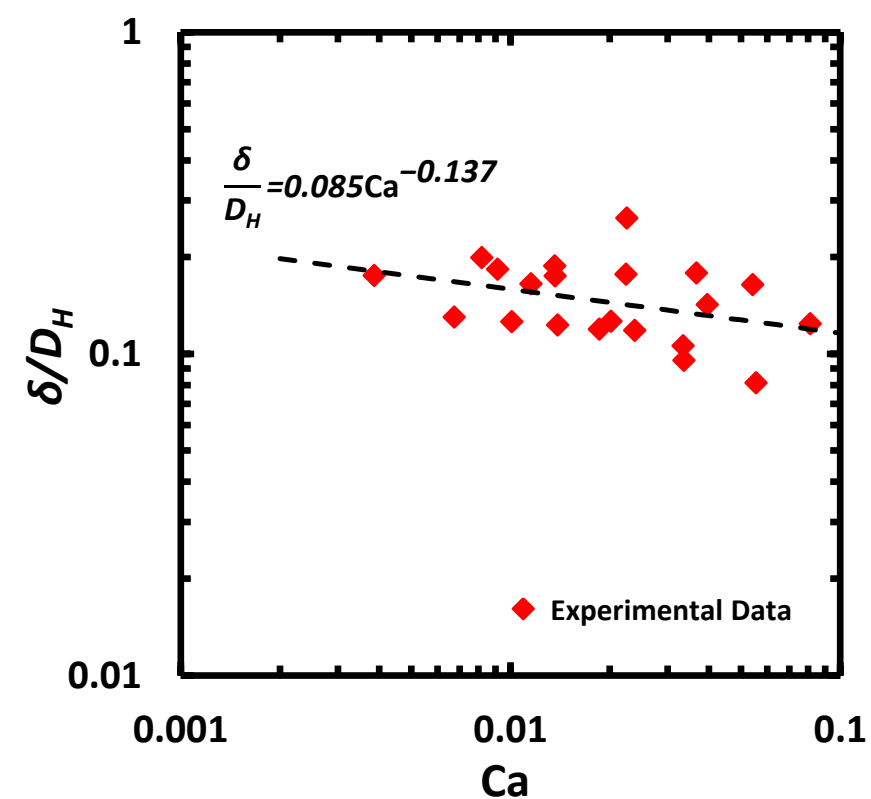

Figure 5 Experimental corner film thickness measurements plotted as a function of capillary number with a dashed line of best fit. 

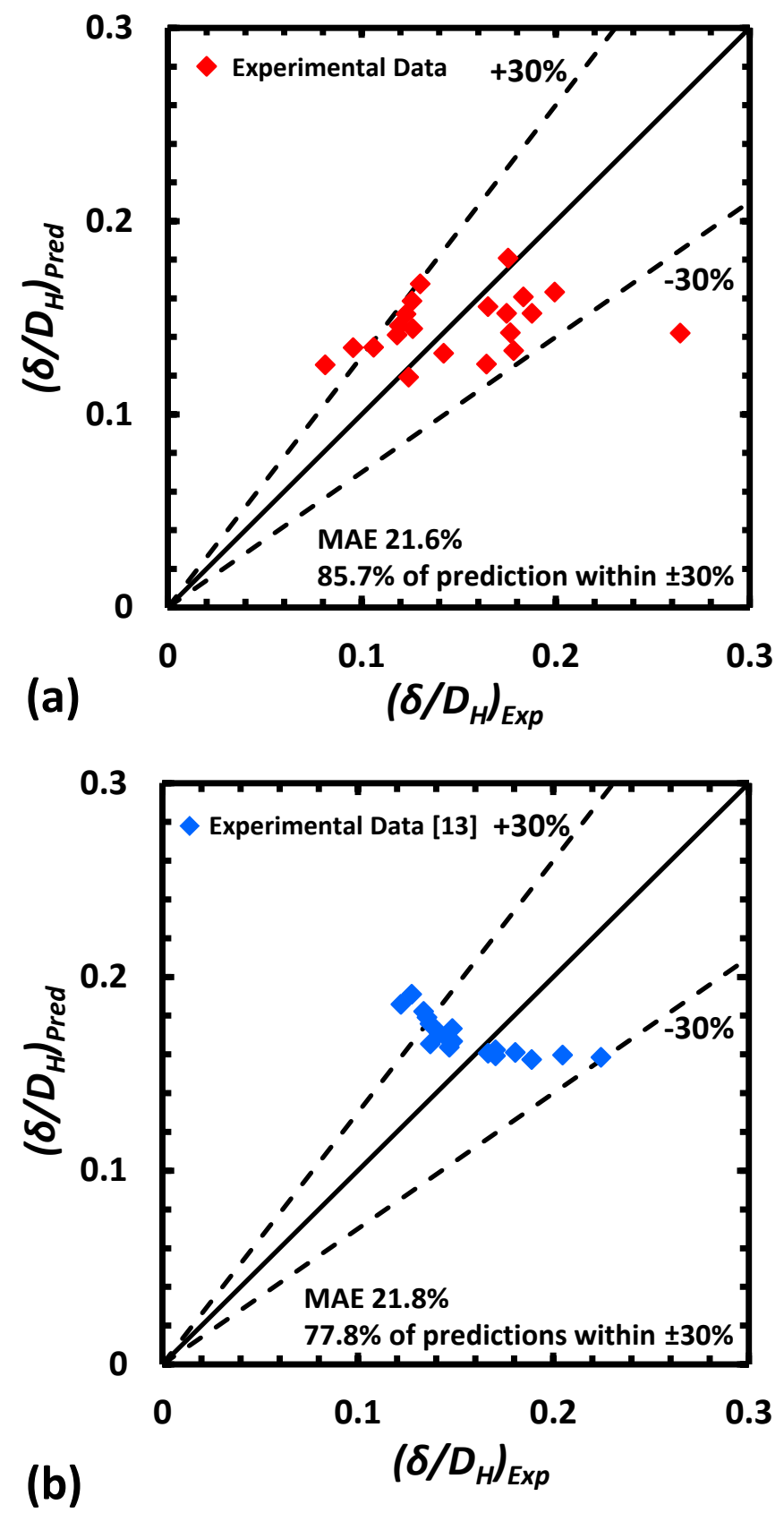

Figure 6 Comparison between experimentally measured film thickness values and predicted values using model developed in present study for data obtained in (a) the parametric experimental investigation presented here and (b) by Chaoqun et al. [13]. 